\title{
Late infantile autosomal recessive myotonia, mental retardation, and skeletal abnormalities: a new autosomal recessive syndrome
}

\author{
ANTONIO RICHIERI-COSTA*, SONIA MARIA GARCIA DA SILVA $\dagger$, AND \\ OSWALDO FROTA-PESSOA* \\ From * Laboratorio de Genetica Humana, Departamento de Biologia, Universidade de Sao Paulo, Sao Paulo; \\ and $\nmid$ Faculdade de Medicina de Botucatu, Departamento de Neurologia, \\ Universidade Estadual Julio de Mesquita Filho, Botucatu, SP, Brazil.
}

SUMMARY Four sibs of non-consanguineous parents who had myotonia from late infancy are described. Mild to moderate mental retardation, severe bone abnormalities of the vertebral column (mainly in the thoracolumbar region), and short stature were also observed. Autosomal recessive inheritance is demonstrated. These cases are compared with reported cases of the Schwartz-Jampel syndrome.

Myotonia is part of the clinical picture of some genetically determined syndromes, such as autosomal dominant myotonia congenita (Thomsen's disease), autosomal recessive myotonia congenita (Becker's syndrome), dystrophia myotonica (Steinert's disease), paramyotonia congenita, and chondrodystrophic myotonia (Schwartz-Jampel syndrome). In this latter condition it is associated with skeletal abnormalities, peculiar facies, and short stature, among other manifestations. ${ }^{12}$ This paper describes four sibs born to a non-consanguineous couple. They have had myotonia since late infancy, mild to moderate mental retardation, skeletal abnormalities mainly in the thoracolumbar vertebral bodies, and short stature. Facial and joint signs are absent.

Received for publication 16 February 1983. Accepted for publication 8 July 1983.
The pedigree data are consistent with an autosomal recessive pattern of inheritance. A clinical comparison is made with the Schwartz-Jampel syndrome. Some of the manifestations observed support the assumption that the syndrome described here is a distinct autosomal recessive disorder.

\section{Case reports}

CASE 1

AG (fig 1 and 2), the proband, is a 15-year-old male, born of a non-consanguineous marriage, after a term pregnancy and uneventful delivery. $\mathrm{He}$ is the seventh of nine sibs. Three other sibs present the same clinical picture and will be described below. The other five sibs are normal.

The proband's neuropsychological development

I

II

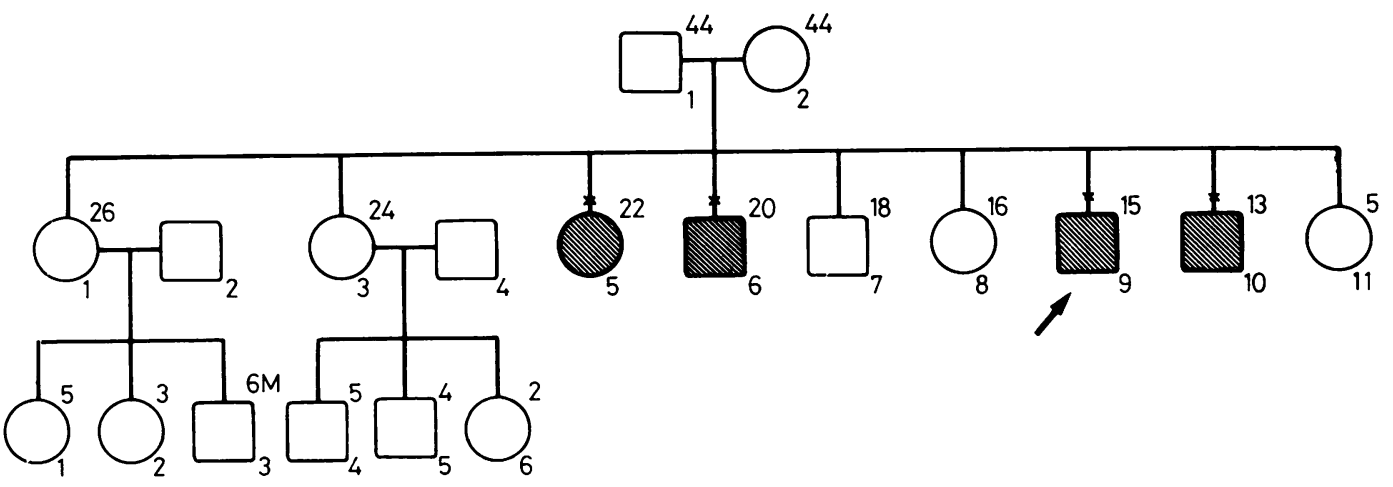

FIG 1 Family pedigree. $x=$ examined by the authors. 


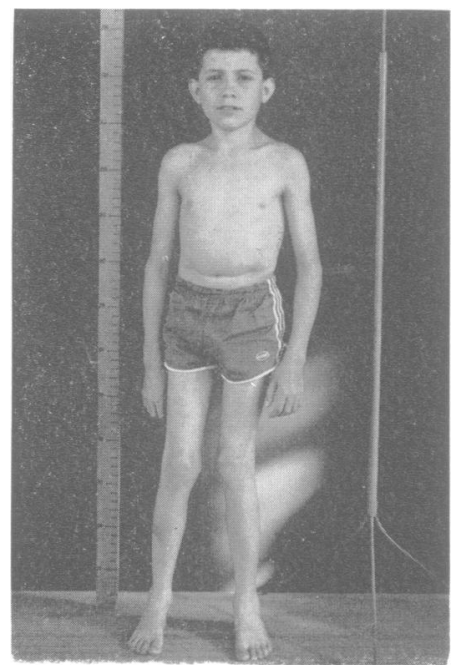

FIG 2 Front view of case 1.

has been delayed, mainly in relation to mental skills. $\mathrm{He}$ is presently an out-patient in an institution for mentally handicapped children. His neuromotor development was normal until he was 5 years old when he started having 'painful spasms' in the legs. At 6 years of age he started having difficulty in swallowing solid food, accompanied occasionally by pain. From 7 years of age onwards, his gait became progressively stiff. When he was 11 , alterations were noticed in his chest, as well as in the alignment of his vertebral column. His voice became snuffly and the 'painful spasms' in the lower limbs became more intense. His clinical picture has progressed slowly and he now has a rigid gait.

His height is $133 \mathrm{~cm}$ (below the 3rd centile) and the head circumference is $53.5 \mathrm{~cm}$ (normal). He has short stature, pectus carinatum, and generalised muscular hypertrophy with a firm consistency. A stiff gait with no joint limitation, normal deep tendon reflexes, and slightly decreased strength in the lower limbs are also present. Cranial nerve and sensory examination was normal. Myotonia is spontaneously present in the muscles of the arm, forearm, and scapular girdle and it can be elicited by percussion of all voluntary muscles except the facial and ocular muscles. Action myotonia is visible in the hands after repetitive opening and closing. Kyphoscoliosis and mental retardation are present. Cardiac, pulmonary, abdominal, and genital examinations were normal.

Routine blood and urine tests were normal. Serum levels of $\mathrm{Na}, \mathrm{K}, \mathrm{P}$, and $\mathrm{Ca}$ were also normal, as were SGOT, SGPT, and C.K. Screening tests for metabolic defects showed no abnormalities.
EKG was normal. On the EMG, electrical activity showed some silent periods mixed with high frequency discharge periods at rest. The motor unit potentials showed a myopathic pattern. Myotonic discharges were present in all muscles examined. Sensory and motor conduction both showed normal velocity (fig 3 ).

Histological examination showed focal areas where the muscle fibres were broken and displayed an irregular outline. These areas lacked fibre striation and had a hyaline aspect. No inflammatory reaction was observed.

Generalised bone demineralisation was seen on $x$-ray, except in the skull and long bones which looked normal. There was mild metatarsus varus bilaterally. There were marked changes in the thoracolumbar vertebral bodies, the main findings being observed in the 1st and 2 nd lumbar vertebrae, which were shortened anteroposteriorly and had a wedge aspect (fig 4). The entire thoracolumbar segment of the spine showed marked irregularities on the surface of the vertebral bodies. Pectus carinatum was prominent. The articular surfaces of the acetabulum and the femoral epiphysis were also irregular.

\section{CASE 2}

DG (fig 1) is a 13 year old male, born after a normal term pregnancy and an uneventful vaginal delivery. Except for delayed mental development, his neuromotor development was normal until the age of 7 . At that time he started having 'painful spasms' in the lower limbs and his gait became progressively stiffer. At 8 years of age, a lateral deviation in the alignment of the vertebral column was noticed. When 9 years old, he developed a snuffly voice, pain and difficulty in swallowing, and his chest became progressively bulging. He now has a stiff

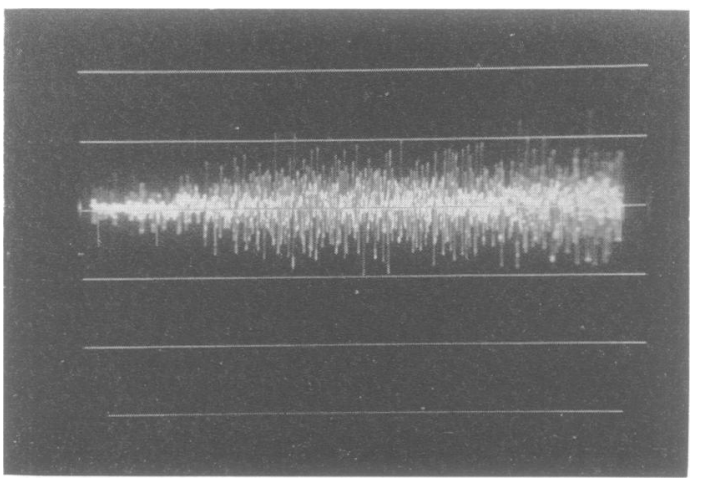

FIG 3 Electromyogram of case 1. 


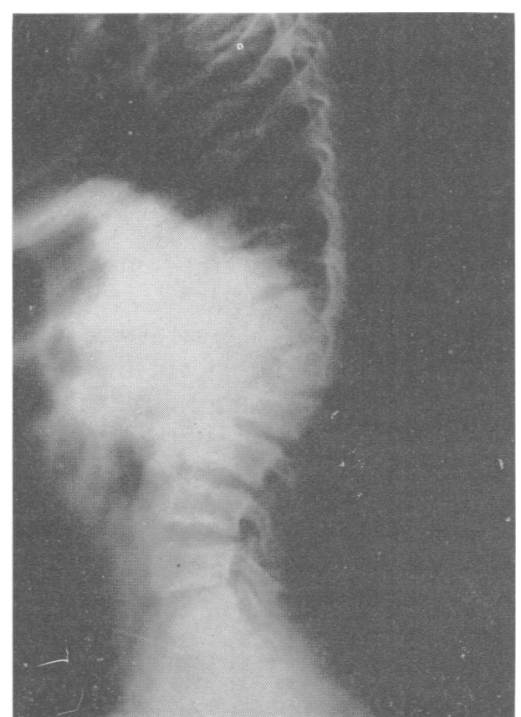

FIG 4 Lateral $x$-ray of the thoracolumbar vertebrae of case 1 .

gait and occasionally falls after suffering sudden generalised spasms.

His height is $131 \mathrm{~cm}$ (below the 3rd centile) and the head circumference is $52 \mathrm{~cm}$ (normal). Short stature, pectus carinatum, and pronounced muscular hypertrophy in the upper limbs are present. The muscles of the lower limbs show mild hypertrophy, but their consistency is quite firm. A stiff gait with no joint limitation, normal tendon reflexes, and slightly decreased strength in the lower limbs are also present. Cranial nerve and sensory examination were normal. During examination he had sudden and generalised spasms, which were interpreted as intention myotonia; this finding was also recorded during the electromyographic study. Myotonia is spontaneously present and can also be elicited by percussion of all voluntary muscles, except the facial and ocular muscles. Action myotonia is visible in the hands after repetitive opening and closing. Kyphoscoliosis and mental retardation are present. Cardiac, pulmonary, abdominal, and genital examinations were normal.

Routine blood and urine tests were normal. Serum levels of $\mathrm{Na}, \mathrm{K}, \mathrm{P}$, and $\mathrm{Ca}$ were also normal, as were SGOT, SGPT, and CK. Screening tests for metabolic defects did not reveal any abnormality.

EKG was normal. The EMG findings were the same as those observed in case 1. Striation of muscle fibres was preserved. Some of the fibres displayed a mild retraction, probably as a result of technical artefacts.

Generalised bone demineralisation was seen on $x$-ray. The 2 nd lumbar vertebra was shortened in its AP aspect. The anterior portion of the thoracolumbar vertebral bodies showed irregularities which gave them a 'step-like' appearance. Pectus carinatum was observed and the articular surfaces of the acetabulum and femoral epiphysis were irregular.

CASE 3

JAG (fig 1) is a 20 year old male, born after a normal term pregnancy and an uneventful vaginal delivery. His neuropsychological development was delayed. He started to walk when he was 30 months old. Since then he has had weakness in the lower limbs associated with frequent falls. At 5 years of age, his movements were noticed to be slow and his gait became progressively stiff. At the same time, the patient started complaining of 'painful spasms' in the muscles of the lower and upper limbs, mainly after exertion. At 8 years of age, alterations in the alignment of the vertebral column and in the aspect of his chest were observed.

His height is $139 \mathrm{~cm}$ (below the $3 \mathrm{rd}$ centile) and the head circumference is $52.5 \mathrm{~cm}$ (normal). He has short stature and a bell-shaped chest. The muscles of his upper limbs show considerable hypertrophy. His gait is stiff but there is no joint limitation. However, he has genu valgum and his thighs lie in mild adduction, bilaterally. His strength is normal, but his tendon reflexes are reduced. Cranial nerve and sensory examination was normal. Myotonia is spontaneously present in the muscles of the upper limbs and scapular girdle. It can be elicited by percussion in all voluntary muscles, except for the facial and ocular muscles. Action myotonia is visible in the hands after repetitive opening and closing. Kyphoscoliosis and mental retardation are present. Cardiac, pulmonary, abdominal, and genital examinations were normal.

Laboratory blood tests were normal, as was the EKG. EMG findings were the same as those observed in case 1. Muscle biopsy was not performed.

Generalised bone demineralisation was seen on $x$-ray. The skull and long bones were normal and there was mild equinovarus of the thighs. The 12th thoracic vertebra was extremely shortened in height and had the shape of a rudimentary wedge. The $3 \mathrm{rd}$ lumbar vertebra was also shortened in the AP aspect but was not wedge-shaped. The surface of the vertebral bodies showed several irregularities and there was severe kyphoscoliosis.

\section{CASE 4}

AnG (fig 1) is a 22 year old female, born after a normal term pregnancy and an uneventful vaginal delivery. 
The patient had delayed neuropsychological development. She started walking when she was 3.5 years old. At 6 years of age, she started having 'painful spasms' in the lower limbs and her gait became stiff. By that time alterations were noticed in the alignment of the vertebral column and in her chest. Her clinical picture progressed slowly with continuous worsening of the gait and of the 'painful spasms' in the lower limbs. At the age of 10 , her voice became snuffly.

The patient has short stature, her chest is bellshaped, she is unable to walk, and she has severe stiffness of the lower limbs. Generalised muscular hypotrophy is also present and tendon reflexes are absent. Although evaluation of strength is difficult, it seems to be decreased. Sensitivity is normal. She has a bilateral convergent strabismus. Myotonia is spontaneously present in the muscles of the upper and lower limbs, girdle, and thorax. It can be elicited by percussion in all voluntary muscles, except for the facial and ocular muscles. Action myotonia is visible in the hands after repetitive opening and closing. Severe kyphoscoliosis and mental retardation are present. Cardiac, pulmonary, abdominal, and genital examinations were normal.

Laboratory blood tests and EKG were normal. EMG and muscle biopsy were not performed. Generalised bone demineralisation was seen on $x$-ray. The skull and long bones were normal. The 12 th thoracic vertebra was shortened in height and in the AP aspect. The 1st lumbar vertebra was rudimentary and was wedge-shaped (fig 5). Coarse irregularities were seen on the surface of the vertebral bodies, mainly in the thoracolumbar region. Severe kyphoscoliosis and irregularities of the acetabulum and femoral epiphysis were also present.

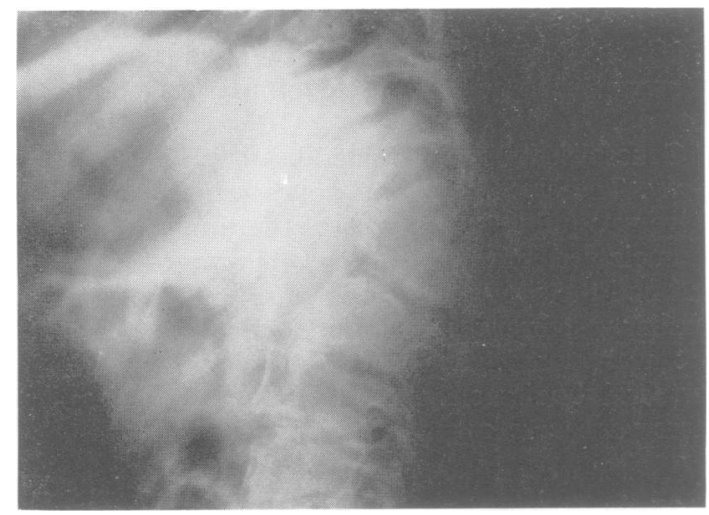

FIG 5 Lateral $x$-ray of the thoracolumbar vertebrae of case 4 .

\section{Discussion}

The clinical picture of this condition is clearly different from that of three known autosomal dominant myotonias (Thomsen's disease, dystrophia myotonica, and paramyotonia congenita) and of the autosomal recessive myotonia congenita. Chondrodystrophic myotonia (Schwartz-Jampel syndrome), however, shows some similarities to the present condition. It is also a rare autosomal recessive disease, characterised by a peculiar facies, myotonia, short stature, multiple skeletal abnormalities, and progressive joint limitation, which starts, in most cases, before the second year of life. ${ }^{1-4}$ The features of the Schwartz-Jampel syndrome are compared with those of the present family in the table. The late onset of the disease, the occurrence of mental retardation, the absence of alterations in facial appearance, and the preferential bone involvement of the thoracolumbar vertebral bodies are the differential signs in the family being studied. Mental retardation has been reported on only two occasions in isolated cases of the Schwartz-Jampel syndrome. Evidence of obstruction of cerebral subarachnoid pathways was present in one of these cases ${ }^{4}$ and absent in the other. ${ }^{2}$

Anomalies found in the Schwartz-Jampel syndrome, such as blepharophimosis, flat face, small mouth, short neck, low set ears, low hairline, high pitched voice, diastasis recti or hernia or both, joint dislocation, joint limitation, and dental alterations, are not present in our family. The clinical picture of

TABLE Frequencies of signs in 19 reviewed cases of the Schwartz-Jampel syndrome $e^{5}$ and in the present family.

\begin{tabular}{|c|c|c|c|c|}
\hline \multirow{2}{*}{ Signs } & \multicolumn{3}{|c|}{ Schwartz-Jampel syndrome } & $\begin{array}{l}\text { Present } \\
\text { family }\end{array}$ \\
\hline & Present & Absent & $\begin{array}{l}\text { Not } \\
\text { stated }\end{array}$ & $\begin{array}{l}\text { Cases } \\
1 \quad 23\end{array}$ \\
\hline
\end{tabular}

Signs present in $75 \%$ or more of the patients Short stature Myotonia

$\begin{array}{lc}\text { ore of the patients } \\ 17 & 0 \\ 15 & 0 \\ 15 & 1\end{array}$
2

Joint limitation

15 +++
$+++t$

Signs present in $50 \%$ to $75 \%$ of the patients Blepharophimosis Pectus carinatum

14

Small mouth

Normal intelligence

Flat face

Short neck

Signs present in less than $50 \%$ of the patients Shortness of vertebrae High pitched voice Muscular hypertrophy Muscular hypotrophy Low set ears

Diastasis recti or hernia

Low hairline

Joint dislocation

Mental retardation

Dental anomalies

\begin{tabular}{cc}
$0 \%$ & of the patients \\
9 & 4 \\
8 & 0 \\
7 & 3 \\
5 & 0 \\
6 & 1 \\
6 & 7 \\
5 & 3 \\
3 & 12 \\
2 & 17 \\
3 & 0 \\
\hline
\end{tabular}


the Schwartz-Jampel syndrome usually starts in the first 2 years of life, whereas in the family presented here the initial manifestations appeared at a mean age of $5 \cdot 7$ years.

From the radiological point of view, the SchwartzJampel syndrome may show one of three patterns: (1) complete absence of skeletal abnormalities, ${ }^{6}$ (2) skeletal alterations without vertebral involvement, 37-9 (case 1) and (3) skeletal abnormalities with vertebral involvement. ${ }^{2} 459$ (case 2)-11 The latter pattern is observed in the family presented here. However, lack of involvement of long bones provides a basis for differentiation.

The reduction in size and irregularities of the vertebral bodies (mainly in the lower thoracic and upper lumbar vertebrae) resemble alterations seen in the mucopolysaccharidoses. However, this condition can be ruled out if we consider the myotonia and other clinical manifestations, as well as the results of laboratory tests observed in the family presented.

The occasional generalised spasms observed in one of the patients were interpreted as intention myotonia, and the swallowing problems in two of them were regarded as a result of myotonic manifestations in their pharyngeal muscles.

A syndromic delineation of the manifestations presented by the family studied can be established as follows. The disorder is a progressive condition, with onset usually at a mean age of 5.7 years, and includes myotonia, progressive impairment of the gait, alterations in the appearance of the chest and in the alignment of the vertebral column, short stature, and mild to moderate mental retardation. The preferential involvement of the thoracolumbar segment of the spine and the morphology of the vertebral bodies are probably primary, but the possibility that these may be related to the muscular disorder must be kept in mind. The clinical signs show no significant differences among the affected patients. As far as clinical prognosis is concerned, the oldest patient, who is 22 years old, presents severe impairment and her clinical prognosis is very poor.
The pedigree analysis shows a pattern consistent with autosomal recessive inheritance in that the condition occurred in four sibs of both sexes, while the parents and other relatives are normal. There is no parental consanguinity.

The authors are grateful to Dr Armando Carrasco for his assistance and advice in this study.

\section{References}

${ }^{1}$ Schwartz O, Jampel RS. Congenital blepharophimosis associated with a unique generalized myopathy. Arch Ophthalmol 1962;68:52-7.

2 Aberfeld DC, Hinterbuchner LP, Schneider M. Myotonia, dwarfism, diffuse bone disease and unusual ocular and facial abnormalities (a new syndrome). Brain 1965;88: 313-22.

3 Mereu TR, Porter IH, Hug G. Myotonia, shortness of stature and hip dysplasia. Schwartz-Jampel syndrome. Am J Dis Child 1969;117:470-8.

4 Huttenlocher PR, Landwirth J, Hanson V, Gallacher BB, Bensch K. Osteo-chondro-muscular dystrophy. A disorder manifested by multiple skeletal deformities, myotonia, and dystrophic changes in muscle. Pediatrics 1969;44: 945-58

5 Mustacchi Z, Enge LGHB, Richieri-Costa A, FrotaPessoa O. Schwartz syndrome associated with teeth and cardiac involvement. Rev Bras Genet 1983;1:93-9.

6 Saadat MH. Schwartz syndrome: myotonia with blepharophimosis and limitation of joints. J Pediatr 1972;81: 348-50.

7 Aberfeld DC, Namba T, Vye MV, Grob D. Chondrodystrophic myotonia: report of two cases. Myotonia, dwarfism, diffuse bone disease and unusual ocular and facial abnormalities. Arch Neurol 1970;22:455-62.

8 Cordeiro-Ferreira N, Gomes da Costa MG, Marques DA. Sindrome de Schwartz. Bord Med 1973;6:1777-86.

9 Fowler WM, Layzer RB, Taylor RG, et al. The SchwartzJampel syndrome: its clinical, physiological and histological expression. $J$ Neurol Sci $1974 ; 22: 127-46$.

10 Beighton P. The Schwartz syndrome in Southern Africa. Clin Genet 1973;4:548-55.

11 Van Huffelen AC, Gabreels FJM, Van Luypen JS, et al. Chondrodystrophic myotonia. Neuropaediatrie 1974;5: 71-90.

Correspondence and requests for reprints to $\mathrm{Dr}$ Antonio Richieri-Costa, Laboratório Genética Humana, Departamento de Biologia, Universidade de São Paulo, CP 11461, 05421 São Paulo, SP, Brazil. 